\title{
Adenoid cystic carcinoma of the Bartholin gland is not HPV-related: A case report and review of literature
}

\author{
Gatske M. Nieuwenhuyzen-de Boer ${ }^{\mathrm{a}}$, Shatavisha Dasgupta ${ }^{\mathrm{b}}$, Patricia C. Ewing-Graham ${ }^{\mathrm{b}}$, \\ Mieke R. Van Bockstal ${ }^{\mathrm{b}, \mathrm{c}, *}$ \\ a Department of Gynaecologic Oncology, Erasmus MC, University Medical Centre Rotterdam, Rotterdam, The Netherlands \\ ${ }^{\mathrm{b}}$ Department of Pathology, Erasmus MC, University Medical Centre Rotterdam, Rotterdam, The Netherlands \\ ${ }^{\mathrm{c}}$ Department of Pathology, University Clinics Saint-Luc, Brussels, Belgium
}

\section{A R T I C L E I N F O}

\section{Keywords:}

Vulva

Bartholin gland

Adenoid cystic carcinoma

Human papilloma virus

Lung metastasis

p16

\begin{abstract}
A B S T R A C T
Adenoid cystic carcinoma (ACC) of the Bartholin gland is a rare gynaecological entity. Despite its slow growth and inconspicuous presentation, vulvar ACC has a propensity for perineural invasion and is therefore associated with high local recurrence rates.

We report a case of vulvar ACC in a 61-year-old woman with a prolonged swelling of the Bartholin gland. This patient presented with pulmonary metastases at the moment of histological diagnosis. The vulvar and the pulmonary lesions showed identical histology. Despite a history of human papilloma virus (HPV)-related usual type vulvar intra-epithelial neoplasia and cervical squamous cell carcinoma, the vulvar ACC was negative for both p16 immunohistochemistry and HPV-DNA.

We conclude that HPV is not involved in the pathogenesis of pure ACC of the Bartholin gland. Additionally, we advocate a low threshold for performing biopsies of vulvar swellings in women aged $>40$ years, to rule out malignancy and to prevent diagnostic delays.
\end{abstract}

\section{Introduction}

Adenoid cystic carcinoma (ACC) is a rare variant of an adenocarcinoma, which can arise in any organ with seromucinous or sweat glands. Although most common in the major and minor salivary glands, ACC has also been reported to occur in the skin, the lacrimal glands, the breast, the oesophagus, the upper and lower respiratory tract and the female genital tract [1-3]. In the vulva, ACC usually originates from the Bartholin gland [3]. Primary carcinomas of the Bartholin gland account for less than $5 \%$ of all vulvar carcinomas and less than $1 \%$ of all female genital tract malignancies [4,5]. ACC represents around $30 \%$ of Bartholin gland tumours and is therefore a rare gynaecological entity [4]. Although slowly growing, vulvar ACC is notorious for its high local recurrence rates, due to the propensity for extensive perineural invasion [4]. Because of its rarity, slow growth and non-specific symptoms, the possibility of an ACC is often not considered and therefore the diagnosis is frequently delayed. Here, we report a case of vulvar ACC in a 61-yearold woman with a history of human papilloma virus (HPV)-related disease. We provide a brief overview of the relevant literature regarding the association between ACC and HPV, and we discuss the lessons learnt from this case.

\subsection{Case history}

A 61-year-old woman presented with pain in the right vulvovaginal area. Upon clinical examination, a mobile, tender swelling of less than one centimetre in size was detected on the right labium majus. The skin overlying the vulvar swelling was intact. A provisional diagnosis of a cyst of the Bartholin gland was established. The patient had a history of a squamous cell carcinoma of the cervix (T2bN0) and usual type vulvar intra-epithelial neoplasia (uVIN). Both of these lesions showed diffuse, strong block-positivity with p16 immunohistochemistry, establishing an association with HPV. The cervical squamous cell carcinoma had been treated with a conisation and chemoradiotherapy. Topical imiquimod had been administered for the uVIN lesion. The patient had been disease-free for four years.

After the initial presentation with vulvar pain, the patient was followed up for ten months. Finally, a simple excision of the right Bartholin gland was performed because of persistent pain and protracted vulvar swelling. A poorly circumscribed solid mass was removed, devoid of any cysts. Histopathological examination of the specimen revealed an ACC with a predominantly cribriform growth pattern and extensive perineural invasion (Fig. 1A-1B). Lympho-vascular space

\footnotetext{
* Corresponding author at: Mieke Van Bockstal, Department of Pathology, University Clinics St-Luc, Avenue Hippocrate 10, 1200 Brussels, Belgium.

E-mail addresses: m.vanbockstal@erasmusmc.nl, mieke.vanbockstal@uclouvain.be (M.R. Van Bockstal).
} 


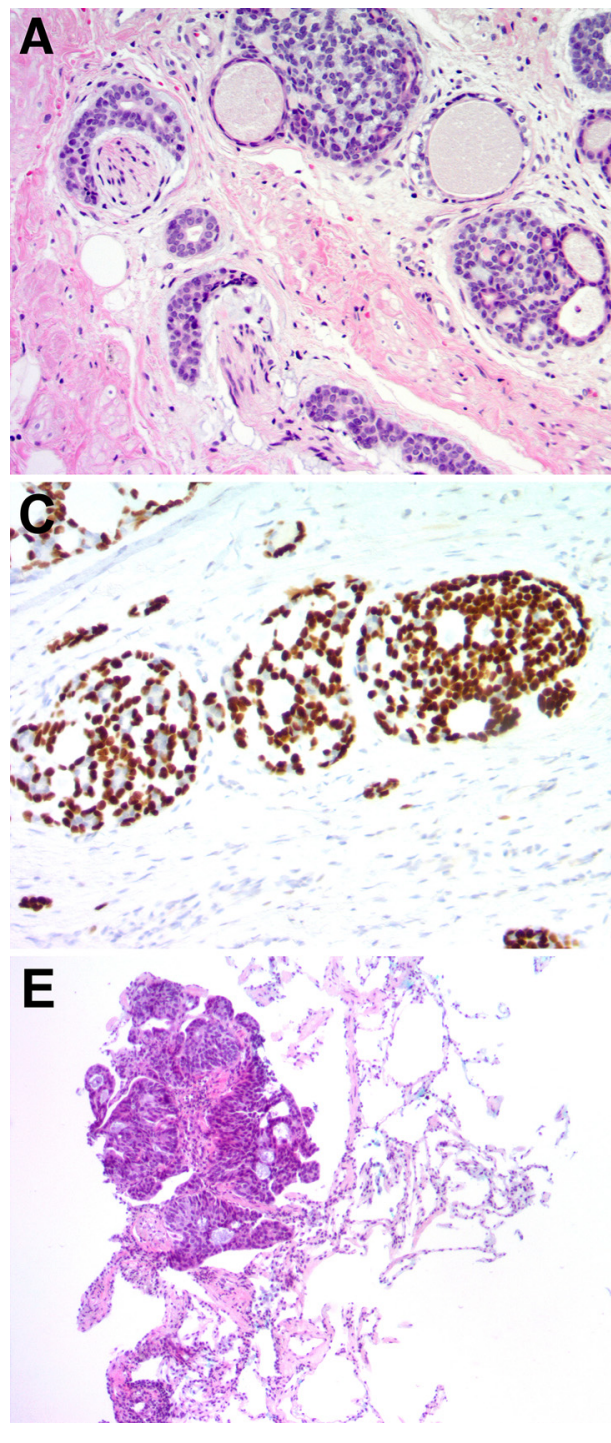

invasion was not observed. The tumour consisted of small glandular lumens and larger pseudo-glandular spaces filled with periodic acidSchiff-positive basement membrane material. The lining cells of these glandular spaces had predominantly monomorphic nuclei, with scant mitotic figures. Both the glandular and pseudo-glandular spaces were surrounded by bland-looking myoepithelial cells, which stained positive with p63 immunohistochemistry (Fig. 1C). Normal glandular structures could not be identified in the excision, and the entire Bartholin gland seemed to be replaced by the tumour.

Since this patient had a history of HPV-related gynaecological disease, p16 immunohistochemistry and molecular HPV-genotyping $\left(\mathrm{SPF}_{10}\right.$-Deia/LiPA, Laboratory BioMedical Products, Rijswijk, The Netherlands) were performed. The ACC showed patchy non-diffuse p16-staining (Fig. 1D), which was regarded as non-specific. The HPVpolymerase chain reaction was also negative. Histologically, the excision was incomplete. Targeted next-generation sequencing did not reveal any potentially pathogenic mutations in mutation hotspots in AKT1 (exon 3), ALK (20, 22-25), APC (14), ARAF (7), BRAF (11, 15), CTNNB1 (3, 7, 8), EGFR (18-21), EZH2 (16), FBWX7 (9, 10), FOXL2 (1), FGFR1 (4, 7, 12), FGFR2 (7, 9, 12), FGFR3 (7, 9), GNA11 (4, 5), GNAQ (4, 5), GNAS (8, 9), HER2 (19-21), HRAS (2-4), IDH1 (4), IDH2 (4), KIT (8, 9, 11, 13, 14, 17), KRAS (2-4), MAP2K1 (2, 3), MET (2, 14, 19), MYD88 (5), NOTCH1 (26, 27), NRAS (2-4), PDGFRA (12, 14, 18), PIK3CA (10, 21), POLD1 (12), POLE (9, 13), RAF1 (7), RET (11, 16), RNF43 (3, 4, 9), ROS1 (38, 41), SMAD4 $(3,9,12)$ and $\operatorname{STK} 11(4,5,8)$.
Fig. 1. Adenoid cystic carcinoma of the Bartholin gland (A-D) with associated pulmonary metastasis (E-F). A. Cribriform growth pattern with extensive perineural invasion (haematoxylin/eosin (HE) stain, original magnification 100x). B. Dual cell population within ACC (HE stain, original magnification 200x). C. Myoepithelial cells staining positive for p63 (original magnification 100x). D. Patchy p16 positivity in the ACC (original magnification 100x). E. Biopsy from a lung nodule showed a morphology similar to the vulvar ACC (original magnification 40x). F. Immunohistochemistry for TTF-1 is negative in the metastasis and positive in the surrounding lung tissue (original magnification 100x).
The genes CDKN2A, PTEN and TP53 were also sequenced, with a coverage of $98 \%, 94 \%$ and $100 \%$, respectively, and did not reveal any mutations.

A dissemination test by computed tomography showed three nodular lesions (size $12-14 \mathrm{~mm}$ ) in the lungs (Fig. 2). One peripherally located pulmonary lesion was biopsied, which showed a tumour with similar morphology to the vulvar ACC. Immunohistochemistry for thyroid transcription factor 1 (TTF-1) was negative (Fig. 1E-F). Given the initial presentation of a vulvar swelling with intact overlying skin, the morphological similarities between the vulvar and the pulmonary lesions, the complete replacement of the Bartholin gland by the tumour, and the lack of evidence for a primary ACC elsewhere, the final diagnosis of a primary Bartholin gland ACC with pulmonary metastasis was established.

\section{Discussion}

This case history illustrates that an early diagnosis of ACC of the Bartholin gland is often hampered by its inconspicuous presentation. Because of the slow growth, the rarity and the lack of specific symptoms, ACCs of the Bartholin gland are often initially mistaken for cysts. Due to the delayed diagnosis, patients with vulvar ACC often present with locally advanced disease [3]. ACC of the Bartholin gland can invade the vaginal wall, ischio-rectal fossa, and even the pubic bone, which often prevents complete surgical removal of the lesion [6]. In 

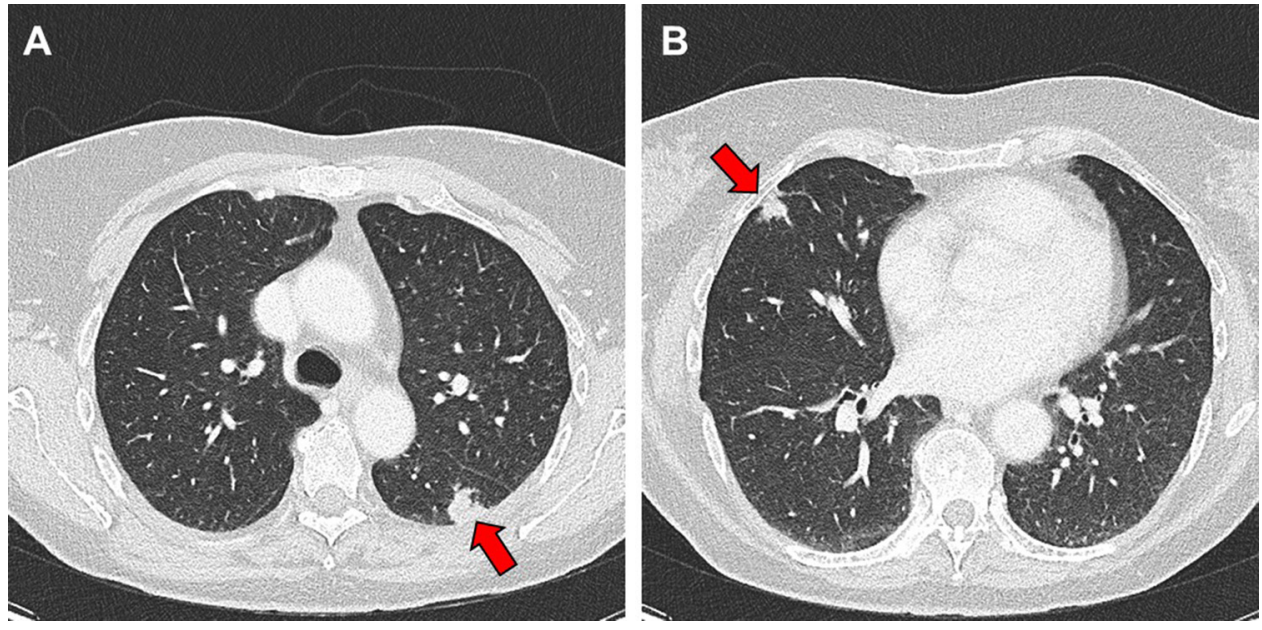

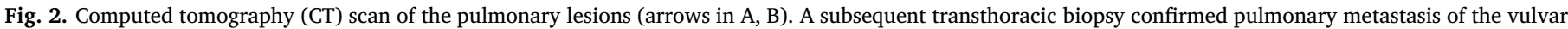
ACC.

retrospect, the vulvar pain reported by this patient was likely caused by perineural invasion.

Despite its rarity, a neoplasm of the Bartholin gland is an important differential diagnosis to consider in women presenting with a Bartholin gland mass, especially when aged over 40 . Most vulvar ACCs occur in postmenopausal women, and reported median age varies from 49 to 59 years $[5,6]$. We therefore advocate a low threshold for performing a biopsy of vulvar swellings in women aged over 40 . In the case of a benign cyst, the contents will be evacuated by the biopsy and symptoms may wane. In the case of a neoplasm, a definite diagnosis will probably be rendered, enabling a wide local excision to minimize the risk of local recurrence. Even when excision appears complete, an ACC is characterized by a high risk of local recurrence, as well as late distant metastases [7]. This is probably due to its tendency for extensive perineural invasion, combined with delayed diagnosis [8]. Where there is local recurrence or inoperable locally advanced disease, radiotherapy can be considered to slow down progression [6].

Patients with ACC of the Bartholin gland generally survive for many years, even in the presence of repeated local recurrence and/or distant metastases $[2,4,6]$. Distant metastases are often pulmonary due to haematogenous spread [2], as was the case in the patient reported herein. Both the vulvar and the pulmonary lesions showed similar histology. The pulmonary lesion did not show nuclear expression of TTF-1. Although rare cases of primary pulmonary ACCs with weak to moderate nuclear TTF-1 staining have been reported, most primary pulmonary ACCs are TTF-1 negative [9]. We therefore consider TTF-1 immunohistochemistry not helpful in the differential diagnosis between primary pulmonary ACC and pulmonary metastases.

The location of the pulmonary lesion(s), however, can aid in this differential, as primary pulmonary ACCs are more commonly located near the trachea or the bronchi than in the pulmonary parenchyma [9]. Since the patient described herein presented with a single vulvar swelling with protracted course, as well as three peripherally located pulmonary lesions, a primary pulmonary ACC with a solitary vulvar metastasis seemed unlikely. We therefore concluded she had a primary vulvar ACC with pulmonary metastases, in concordance with the diagnostic criteria applied by Cardosi et al. [10]. Interestingly, targeted next-generation sequencing did not reveal any potentially pathogenic mutations in mutation hot spots of a panel of commonly mutated genes in many different types of carcinomas. Gain-of-function mutations in the NOTCH1 gene have recently been described as being associated with poor outcome in patients with an adenoid cystic carcinoma [11], but NOTCH1 mutations were lacking in the tumour of this patient.

Since this patient had a history of HPV-related carcinoma, we investigated whether the ACC of the Bartholin gland was HPV-related.
The tumour showed patchy non-diffuse p16-staining and was therefore considered p16-negative. Molecular analysis for HPV was also negative. To our knowledge, pure ACCs of the Bartholin gland are consistently reported to be negative for high risk-HPV $[12,13]$. They present a variable expression of p16, but never diffuse block-positivity [12]. Positivity for p16 and HPV has been described in squamous cell carcinomas or small cell carcinomas of the cervix showing areas of ACC-like differentiation or association with low-grade adenoid basal tumours $[12,14]$. Pure ACCs of the cervix, however, have been reported to harbour high risk HPV [15].

Associations with HPV have also been reported for carcinomas with ACC-like differentiation of the sinonasal tract, and this entity has been named 'HPV-related carcinoma with ACC-like features of the sinonasal tract' [16]. More recently, this entity was renamed as 'HPV-related multi-phenotypic sinonasal carcinoma', as it was acknowledged that the histological spectrum of this HPV-related lesion is much broader than ACC-like morphology alone [17]. Pure ACCs from the Bartholin gland and elsewhere, are known to harbour translocations of the MYB, MYBL1 and NFIB genes, resulting in the novel oncogenic fusion proteins $M Y B$ NFIB and MYBL1-NFIB [13,16]. Unfortunately, these molecular analyses are not available at our centre and therefore this information is lacking for the ACC reported herein. Interestingly, these translocations have not been observed in 'HPV-related multi-phenotypic sinonasal carcinomas', indicating that these tumours have a different pathogenesis than 'true' ACCs and represent a distinct entity [16].

\section{Conclusion}

The unexpected diagnosis of an ACC of the Bartholin gland in a 61year-old illustrates that vulvar swellings in women over 40 should prompt a biopsy to rule out a malignancy. Since carcinomas may mimic a benign cyst of the Bartholin gland, a low threshold for performing biopsies could prevent delays in diagnosis. Additionally, this case corroborates the hypothesis that HPV does not play a role in the pathogenesis of pure ACC of the Bartholin gland.

\section{Compliance with ethical standards}

A written informed consent was obtained from the patient for the publication of this case report, as well as for the accompanying images, as per local protocol.

\section{CRediT authorship contribution statement}

Gatske M. Nieuwenhuyzen-de Boer: Data curation, Methodology, 
Writing - review \& editing. Shatavisha Dasgupta: Formal analysis, Methodology, Software, Visualization, Writing - review \& editing. Patricia C. Ewing-Graham: Formal analysis, Methodology, Writing review \& editing. Mieke R. Van Bockstal: Conceptualization, Investigation, Formal analysis, Writing - original draft.

\section{Declaration of Competing Interest}

No funding was received for this report. All authors declare that they do not have any competing interests.

\section{Acknowledgements}

The authors thank the patient reported in this manuscript for her written informed consent. The authors are grateful to dr. H.C. van Doorn for critically reading the manuscript.

\section{Appendix A. Supplementary data}

Supplementary material related to this article can be found, in the online version, at doi:https://doi.org/10.1016/j.prp.2020.152968.

\section{References}

[1] H. Hametoja, K. Hirvonen, J. Hagstrom, et al., Early stage minor salivary gland adenoid cystic carcinoma has favourable prognosis, Virchows Arch. 471 (2017) 785-792, https://doi.org/10.1007/s00428-017-2163-6.

[2] L.J. Copeland, N. Sneige, D.M. Gershenson, et al., Adenoid cystic carcinoma of Bartholin gland, Obstet. Gynecol. 67 (1986) 115-120.

[3] L.R. Johnson, R.P. Nair, S. Sambasivan, et al., Adenoid cystic carcinoma of Vulva-11 years' single-institution experience, J. Obstet. Gynaecol. India 67 (2017) 196-201, https://doi.org/10.1007/s13224-016-0935-6.

[4] V. Di Donato, A. Casorelli, E. Bardhi, et al., Bartholin gland cancer, Crit. Rev. Oncol. Hematol. 117 (2017) 1-11, https://doi.org/10.1016/j.critrevonc.2017.06.005.

[5] F.M. Woida, A. Ribeiro-Silva, Adenoid cystic carcinoma of the Bartholin gland: an overview, Arch. Pathol. Lab. Med. 131 (2007) 796-798.

[6] G. Yoon, H.S. Kim, Y.Y. Lee, et al, Analysis of clinical outcomes of patients with adenoid cystic carcinoma of Bartholin glands, Int. J. Clin. Exp. Pathol. 8 (2015) 5688-5694.

[7] A. Obermair, S. Koller, A.J. Crandon, et al., Primary Bartholin gland carcinoma: a report of seven cases, Aust. N. Z. J. Obstet. Gynaecol. 41 (2001) 78-81.

[8] L. Agolli, M.F. Osti, V. Armosini, et al., Adenoid cystic carcinoma of Bartholin's gland receiving adjuvant radiation therapy: case report, Eur. J. Gynaecol. Oncol. 34 (2013) 487-488.

[9] M.M. Hu, Y. Hu, J.B. He, et al., Primary adenoid cystic carcinoma of the lung: clinicopathological features, treatment and results, Oncol Letters. 9 (2015) 1475-1481, https://doi.org/10.3892/ol.2015.2859.

[10] R.J. Cardosi, A. Speights, J.V. Fiorica, et al., Bartholin's gland carcinoma: a 15-year experience, Gynecol. Oncol. 82 (2001) 247-251, https://doi.org/10.1006/gyno. 2001.6304.

[11] D.P. Sajed, W.C. Faquin, C. Carey, et al., Diffuse staining for activated NOTCH1 correlates with NOTCH1 mutation status and is associated with worse outcome in adenoid cystic carcinoma, Am. J. Surg. Pathol. 41 (2017) 1473-1482, https://doi. org/10.1097/PAS.0000000000000945.

[12] D. Xing, J.K. Schoolmeester, Z. Ren, et al., Lower female genital tract tumors with adenoid cystic differentiation: P16 expression and high-risk HPV detection, Am. J. Surg. Pathol. 40 (2016) 529-536, https://doi.org/10.1097/PAS. 0000000000000565.

[13] D. Xing, S. Bakhsh, N. Melnyk, et al., Frequent NFIB-associated gene rearrangement in adenoid cystic carcinoma of the vulva, Int. J. Gynecol. Pathol. 36 (2017) 289-293, https://doi.org/10.1097/PGP.0000000000000324.

[14] A.V. Parwani, A.E. Smith Sehdev, R.J. Kurman, et al., Cervical adenoid basal tumors comprised of adenoid basal epithelioma associated with various types of invasive carcinoma: clinicopathologic features, human papillomavirus DNA detection, and P16 expression, Hum. Pathol. 36 (2005) 82-90, https://doi.org/10.1016/j. humpath.2004.08.015.

[15] W. Grayson, L. Taylor, K. Cooper, Detection of integrated high risk human papillomavirus in adenoid cystic carcinoma of the uterine cervix, J. Clin. Pathol. 49 (1996) 805-809.

[16] S. Andreasen, J.A. Bishop, T.V. Hansen, et al., Human papillomavirus-related carcinoma with adenoid cystic-like features of the sinonasal tract: clinical and morphological characterization of six new cases, Histopathology. 70 (2017) 880-888, https://doi.org/10.1111/his.13162.

[17] J.A. Bishop, S. Andreasen, J.F. Hang, et al., HPV-related multiphenotypic sinonasal carcinoma: an expanded series of 49 cases of the tumor formerly known as HPVrelated carcinoma with adenoid cystic carcinoma-like features, Am. J. Surg. Pathol. 41 (2017) 1690-1701, https://doi.org/10.1097/PAS.0000000000000944. 